\title{
Antagonistic experimental coevolution with a parasite increases host recombination frequency
}

\author{
Niels AG Kerstes ${ }^{1 *}$, Camillo Bérénos ${ }^{1,2}$, Paul Schmid-Hempel ${ }^{1}$ and K Mathias Wegner ${ }^{1,3,4}$
}

\begin{abstract}
Background: One of the big remaining challenges in evolutionary biology is to understand the evolution and maintenance of meiotic recombination. As recombination breaks down successful genotypes, it should be selected for only under very limited conditions. Yet, recombination is very common and phylogenetically widespread. The Red Queen Hypothesis is one of the most prominent hypotheses for the adaptive value of recombination and sexual reproduction. The Red Queen Hypothesis predicts an advantage of recombination for hosts that are coevolving with their parasites. We tested predictions of the hypothesis with experimental coevolution using the red flour beetle, Tribolium castaneum, and its microsporidian parasite, Nosema whitei.

Results: By measuring recombination directly in the individuals under selection, we found that recombination in the host population was increased after 11 generations of coevolution. Detailed insights into genotypic and phenotypic changes occurring during the coevolution experiment furthermore helped us to reconstruct the coevolutionary dynamics that were associated with this increase in recombination frequency. As coevolved lines maintained higher genetic diversity than control lines, and because there was no evidence for heterozygote advantage or for a plastic response of recombination to infection, the observed increase in recombination most likely represented an adaptive host response under Red Queen dynamics.

Conclusions: This study provides direct, experimental evidence for an increase in recombination frequency under host-parasite coevolution in an obligatory outcrossing species. Combined with earlier results, the Red Queen process is the most likely explanation for this observation.
\end{abstract}

\section{Background}

Meiotic recombination breaks down genotypes that have proven to be successful, and therefore it was thought to evolve only under very limited conditions [1]. One hypothesis for the adaptive value of recombination, the Red Queen Hypothesis [2-4], suggests that parasites play an important role in maintaining non-zero recombination rates in their hosts. Parasites are ubiquitous, and parasitism is considered to be one of the most significant selection factors for any organism [5]. According to the Red Queen Hypothesis, hosts and parasites engage in sustained and fluctuating antagonistic coevolution, during which the parasite population continuously adapts to the most common genotypes in the host population [2,3]. Rare host genotypes, by contrast, enjoy

\footnotetext{
* Correspondence: niels.kerstes@env.ethz.ch

'ETH Zürich, Institute of Integrative Biology, Experimental Ecology, CH-8092 Zürich, Switzerland

Full list of author information is available at the end of the article
}

a selective advantage and thus will rise in frequency and become common in turn. The parasite population will continue to adapt to host genotypes that are common, but formerly rare, and thus fuel the sustained, fluctuating coevolutionary process. Because rare resistance genotypes are expected to always have a selective advantage under negative frequency-dependent selection, host genotypes will fluctuate over time and genetic diversity is maintained [6,7]. In sexual organisms, rare genotypes are continuously created by meiotic recombination, either via segregation or chromosomal crossovers. As rare recombinants enjoy a selective advantage, recombination should lead to an increase in the mean fitness of host offspring [8] and thus favour the spread of a (linked) recombination modifier in the population. Although theoretical analyses show that the detailed evolutionary processes that favour such modifiers are somewhat more complex $[9,10]$, the essential expectation of the Red Queen Hypothesis still remains that 
recombination should be selectively favoured under antagonistic host-parasite coevolution.

Mathematical models provide theoretical support for the Red Queen Hypothesis $[6,8]$, even though there is controversy over the general applicability of the hypothesis $[11,12]$. So far, several empirical studies have tested key assumptions and predictions of the Red Queen, or have analysed whether observational data is compatible with the hypothesis [9]. For example, there is evidence for the predictions that parasites maintain genetic variation in their host populations $[13,14]$, that parasites track the most common host genotypes [15-18], and that parasite infectivity for certain host genotypes fluctuates over time [19]. By contrast, the evolution and maintenance of recombination rate is rarely directly addressed, as most tests deal with the question of what favours sexual over asexual reproduction [20,21], without considering how parasitism may change recombination rate within a sexual host population itself. Several studies give circumstantial evidence for the Red Queen Hypothesis by demonstrating an association of parasitism with genotypic variation within a host population. Such an association has been found in a range of hosts with different reproductive modes. For example, host recombination correlated positively with parasite load in the grasshopper Eyprepocnemic plorans [22], and immune genes that directly interact with pathogens cluster in regions of higher recombination frequencies in Drosophila melanogaster [23]. Populations of Caenorhabditis elegans exposed to Bacillus thuringiensis showed changes, such as increased genetic diversity, compatible with the Red Queen Hypothesis [13]. Pathogen-induced stress also directly increased somatic recombination in Arabidopsis, which could actually lead to more variation in the progeny as well, since plants lack a predetermined germ line [24]. Similarly, genotypic diversification of offspring by multiple mating led to higher fitness for the mother in Bombus terrestris in the face of parasitism in the field [25]. The most significant field study to date involving antagonistic coevolution of the freshwater snail, Potamopyrgus antipodarium, a species that has both sexual and asexual populations, with its trematode parasites, shows that sexual (i.e. recombining) snail variants occur more often in environments with high parasite pressure [26,27]. In a recent study, coevolution of $C$. elegans with a bacterial pathogen selected for higher outcrossing rates in mixed mating experimental populations [28]. Direct experimental support for a change in recombination rate compatible with the Red Queen Hypothesis in obligatory outcrossing species has so far only been reported in one of our earlier studies using Tribolium castaneum as the host and Nosema whitei as its parasite [29]. This finding could not be confirmed in a follow-up study [30], yet post-hoc checks suggested that the extant genetic variation in the hosts probably was too small to sustain an adaptive response (unpubl. data).

The Tribolium-Nosema system is an ideal test ground for Red Queen-related questions, since it meets the key assumptions of the hypothesis [31-34]. For example, $N$. whitei is an obligately killing parasite [31], which means that there is a severe fitness cost for parasites that are not able to infect or kill their host. In fact, strong selection on the parasite can favour higher recombination rates in the host, even if selection on the host is weak [10]. Furthermore, there is a substantial epistatic component of resistance of $T$. castaneum to infection with $N$. whitei [32,33]. Epistasis in turn, generates linkage disequilibrium, which can be broken down by recombination, thereby creating fitter genotypes. Finally, host genotype versus parasite genotype interactions are found for $N$. whitei interacting with $T$. castaneum [34].

Here, we compared - relative to uninfected control lines - the recombination rates in four replicate $T$. castaneum lines that were allowed to coevolve with a mixture of eight $N$. whitei isolates. More specifically, recombination frequencies were measured in males from both treatments after eleven discrete generations, using microsatellite markers that bordered ten intervals on the genome, which were distributed over four linkage groups. Using this method enabled us to measure recombination directly in the individuals under selection, as opposed to earlier studies that measured recombination in the offspring of the selected individuals, and provided us with a better genomic coverage than in any previous study $[29,30]$. The experimental design was such that control (no parasite) and coevolution (with parasites) treatments were paired within each of the eight replicate lines of the host. We chose the conditions of the experiment (number of generations, replicate lines, etc.) based on the experience and results gained in previous experiments with the same study system $[29,30]$.

Earlier findings from the same coevolution experiment showed that coevolved host lines maintained higher genetic diversity than control host lines [14]. If genetic diversity in the coevolved lines is maintained because of negative frequency-dependent selection by the parasite, it could be assumed that in every generation rare host genotypes created by recombination enjoy an advantage. When parasite-mediated selection is strong enough, one would thus expect to see higher recombination frequencies evolve in the coevolving beetle lines. Such an increase in recombination frequencies, in combination with the observed maintenance of genetic diversity, can therefore be seen as evidence for the Red Queen Hypothesis in an obligatory outcrossing species. Here, we show that host recombination did indeed increase 
during coevolution with a parasite, and that this change is compatible with fluctuating selection exerted by the parasite.

\section{Results and discussion}

After 11 generations of experimental coevolution, the mean difference between observed and expected Kosambi's map distance in the control lines (without parasites) did not relate to the expected map distance and did not significantly deviate from the line of no difference (Figure 1a). By contrast, there was a significant logarithmic relationship between the mean difference of observed and expected map distance, and the length of the genomic interval (map distance) in the coevolved lines (Figure $1 \mathrm{~b}$ ). In addition, the slope of the coevolution regression line was significantly different from the slope of the control regression line (linear regression, $\beta 3=$ $\left.69.212, t_{13}=3.796, P=0.002\right)$. Although it appears, at first sight, that only short intervals increased in recombination frequency in the coevolution treatment, the longer intervals (right hand side) might still be reconciled with an overall increase in recombination. If the true recombination frequency has increased at the same rate all over the genome, across all intervals, then it will be harder to observe this increase in larger intervals (as illustrated in Figure 1b). In those relatively large intervals, the observed recombination rate is closer to its maximum of $50 \%$, and therefore a similar increase in actual recombination frequency results in a relatively small increase in observed recombination values. Even though we used Kosambi's map function to convert observed recombination values to map distances, any such analysis still suffers from this issue, and changes in the real recombination rate in larger intervals are likely to remain undetected. Furthermore, negative values at larger intervals for both treatments (Figure 1a, b) suggest that there was a systematic bias towards lower recombination rates in the lines used here relative to the expected map distances. This might simply be due to the fact that the standard genetic map of $T$. castaneum [35], from which the expected map distances were derived, was based on different beetle populations than the ones used in this experiment. Presumably, the real "line of no difference" of our data lies below the zero lines in the graphs.

Thirteen direct comparisons could be made between the map distance of the same interval for beetle lines that were paired over control and coevolved treatments (i.e. the same lines having been split and assigned to the two treatments). The direct comparison showed essentially the same pattern as described above, since for the short intervals map distances were higher in the coevolved lines than in the control lines. This difference disappeared with increasing interval size (Figure 2), something that can again be explained by the fact that changes in observed recombination frequency in larger intervals are relatively small and therefore harder to detect. The observed pattern might thus indicate an overall increased recombination frequency.

Showing that host recombination has increased during coevolution with a parasite does not provide unequivocal evidence for the Red Queen Hypothesis. For example directional selection, in combination with the effects of limited population size [36], might alternatively contribute to this result. However, directional selection for insecticide resistance did not result in higher recombination frequencies in this system [29]. Instead, there are several findings suggesting that our result is tied to the process of antagonistic coevolution. For example, coevolving populations maintained higher levels of heterozygosity and allelic diversity than control populations in this experiment [14], which is another prediction based on the Red Queen Hypothesis, and which does not match the scenario of directional selection. At the same time we could show that higher levels of heterozygosity is not per se the reason for higher parasite resistance in $T$. castaneum, as inbreeding does not increase the overall susceptibility of the beetles to parasitic infections (for both our case of $N$. whitei (Kerstes et al., in prep) as well as for infections by the rat tapeworm Hymenolepis diminuta [37]). In a similar fashion, there was no significant difference in $\mathrm{F}_{\mathrm{IS}}$-values (inbreeding coefficient) between coevolved and control populations in this experiment [14], and outcrossed beetles were not more resistant to $N$. whitei than their most resistant parent in general ([32], Kerstes et al., in prep). Furthermore, parasite-induced mortality was shown to fluctuate during the first generations of the coevolution experiment [38]. All these findings combined suggest that genetic diversity is maintained in the host populations not because of heterozygote advantage/overdominance, but likely as a result of fluctuating selection exerted by the parasite.

Since the current stock host populations differ from those used in the earlier experiments that produced comparable patterns [29], it seems highly unlikely that our current results are caused by the presence of a particularly favourable initial linkage disequilibrium between a recombination modifier and a locus under selection from the parasites [29]. Furthermore, the replicate populations were all started from different initial linkage disequilibria. It appears that recombination frequency in populations of $T$. castaneum responds very quickly to selection [39], which suggests that our result is due to actual, adaptive evolutionary change. Indeed, no evidence was found for an infection-induced plastic change in recombination in this system [30].

It is nevertheless conceivable that prolonged exposure to non-coevolving parasites would lead to an increase in 


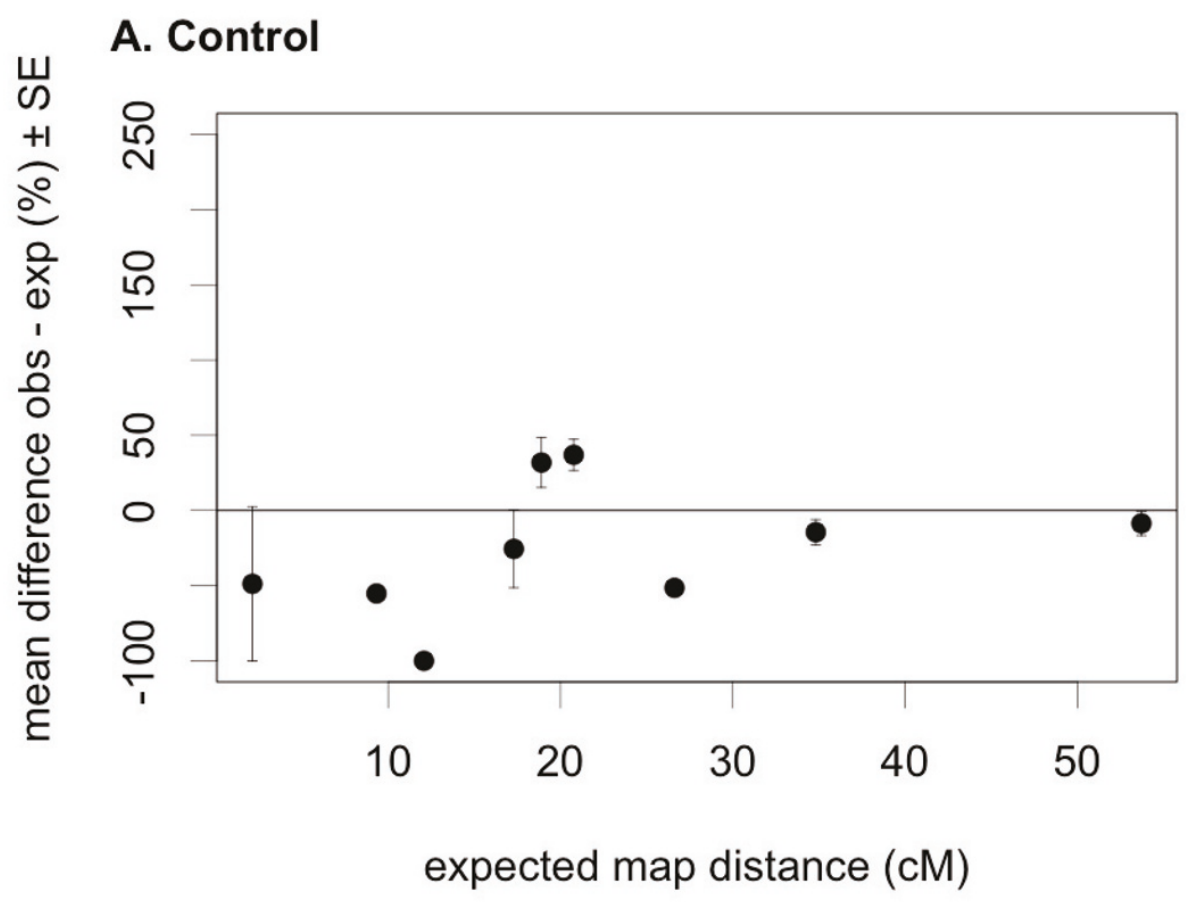

w
+1
0
0
0
0
0
1
0
0
0
0
$\frac{1}{0}$
$\frac{1}{4}$
$\frac{4}{0}$
$\frac{1}{0}$
0
ह

B. Coevolved

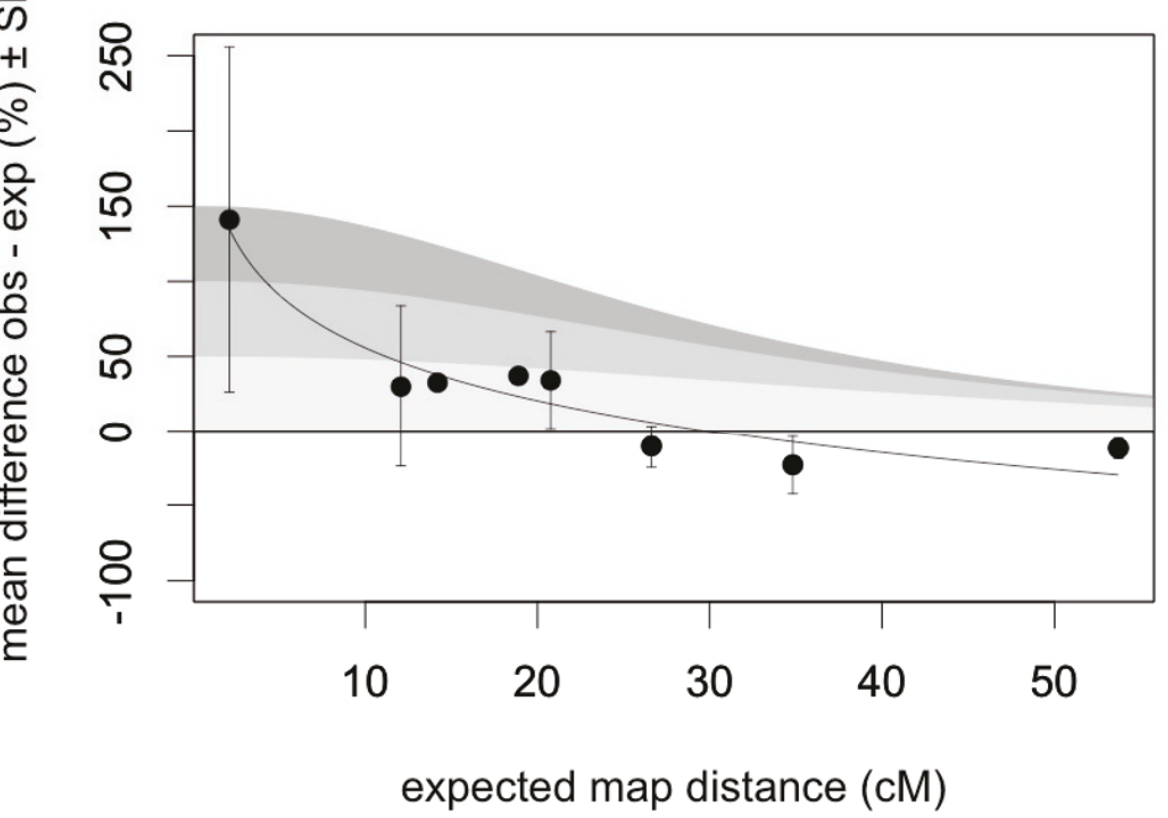

Figure 1 Mean relative difference between observed and expected map distance against the expected map distance. The figure shows all data, i.e. all recombination measurements in all intervals of all lines (35 distinct recombination measurements in control lines, and 24 distinct recombination measurements in coevolved lines). Recombination frequencies were transformed into map distances. Map distances were averaged over interval and line. $\mathbf{a}$, The relative difference does not deviate from a non-difference line and does not correlate with interval length (linear regression, $n=9, F_{1,7}=0.986, P=0.354$ ), suggesting that recombination in the control treatment did not deviate from expected recombination. $\mathbf{b}$, In the coevolving lines a significant logarithmic relationship was observed (linear regression, $n=8, F_{1,6}=64.310, P<0.001, R^{2}$ $=0.915$ ), which might indicate an overall increase in recombination. The grey areas represent the theoretical change in observed recombination rate, in terms of percentage, in the cases of a 50\% (light grey), 100\% (medium grey) and a 150\% (dark grey) genome-wide increase in actual map distance. Kosambi's map function was used to convert map distances into recombination frequencies. The areas illustrate that for large intervals it might be hard to detect a change in recombination frequency, even in the case of a substantial increase in the actual map distance. 


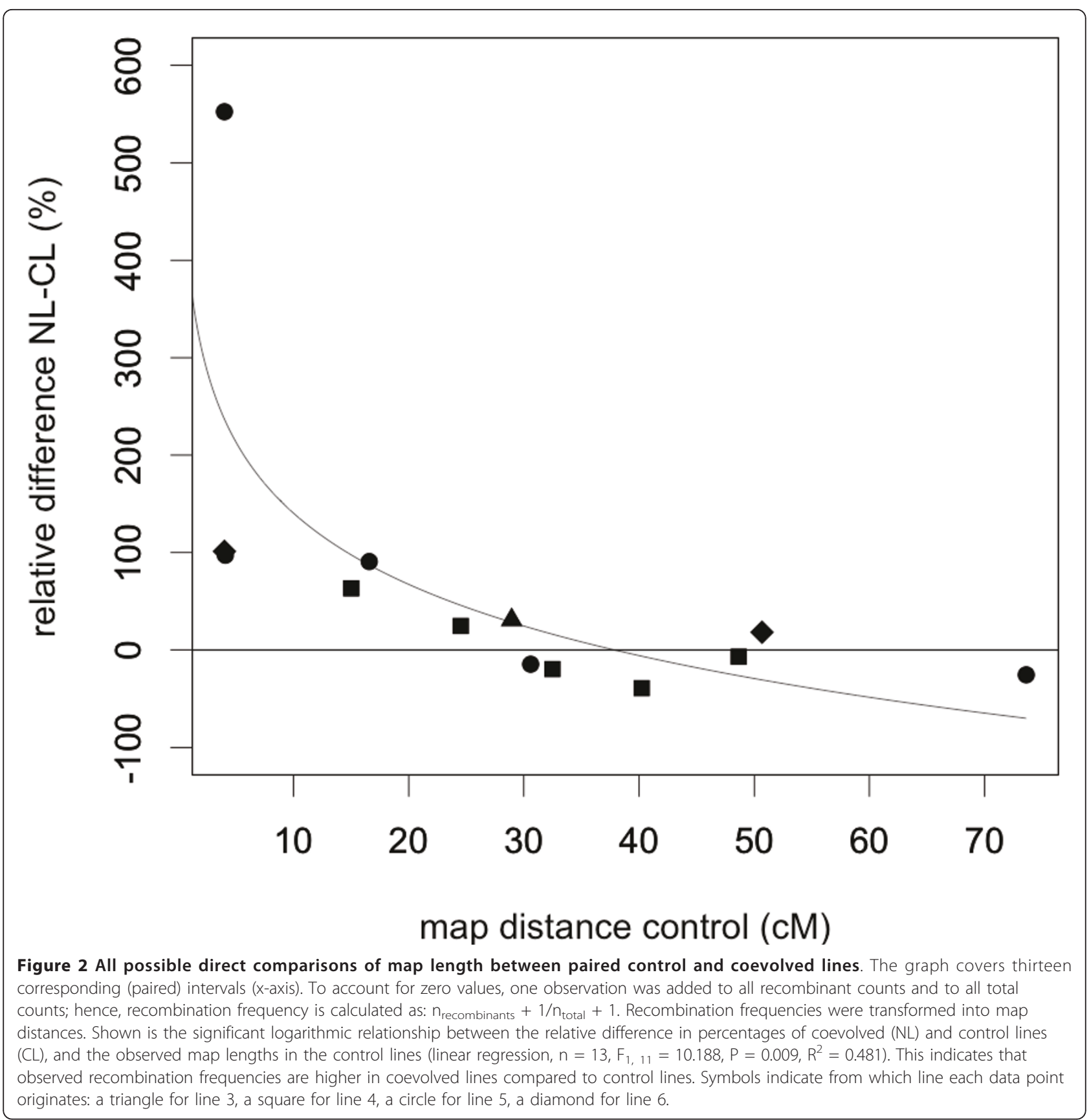

host recombination frequencies, too. However, in a previous study with the same experimental paradigm we found that populations of $T$. castaneum that were exposed to randomly selected, non-evolving, parasites for 12 generations did not differ in recombination rate from controls [29]. Hence, even though we cannot forcefully rule out an effect of simple exposure to parasites in a single all-encompassing experiment, and since there are potentially many other alternative explanations besides fluctuating selection due to the Red Queen process (e.g. genetic correlations between resistance and other traits [40]) to explain the maintenance of genetic diversity and the increase in recombination rate, currently the Red Queen Hypothesis remains the major contender.

We also did observe that host populations became more resistant during coevolution with the parasite [41], contrary to a scenario of pure negative frequency-dependent selection where no such mean change is expected [3]. Host resistance to infection with $N$. whitei has been shown to be a complex trait [32], and one could imagine that some components of resistance can be under 
fluctuating selection, while others are under directional selection. The idea that, in particular during the early phase of the experiment, fluctuating and directional selection were both important fits the observation that parasite-induced mortality was shown to fluctuate during the first generations of the coevolution experiment, while there was a general decrease of parasite-induced mortality (and infectivity) over time, and fluctuations only disappeared in the longer term [38].

The observed decrease in parasite infectivity might indicate that the hosts are ahead in the arms race with their parasites, because a loss in infectivity can only be considered maladaptive for the parasite. We interpret this finding as a possible result of more rapid depletion of genetic variation in the parasite populations than in their coevolving antagonists. As our experimental conditions did not allow for migration between replicate populations, and because $N$. whitei is asexual [42], the parasite population would indeed lose variation that might not be compensated by mutations. At the same time, under our experimental conditions, the benefit of increased recombination frequencies for the host population might disappear in the long term for the same reason.

\section{Conclusions}

We found that, after 11 generations of coevolution, recombination frequency in the host population was increased. Based on insights into genotypic [14] and phenotypic [38] changes that occurred during the coevolution experiment, the observed increase in host recombination frequency is likely to be the result of fluctuating selection exerted by the parasite during early stages of coevolution, although we cannot completely exclude alternative explanations. In this study we benefited from a better genomic coverage, direct recombination measurements, and an improved understanding of the mechanisms behind our observations. We can show that host-parasite coevolution affects the evolution of recombination. Together with the findings of other recent studies [15,28,43], we provide experimental support for the Red Queen Hypothesis as a theory to explain the evolution and maintenance of recombination.

\section{Methods}

\section{Host-parasite coevolution}

All beetles were maintained at $32^{\circ} \mathrm{C}, 70 \%$ humidity, in $24 \mathrm{~h}$ darkness, on standard medium (type 550 'Knospe' organic flour containing $5 \%$ dried yeast). All beetle stock populations used in the experiment were kept at large population sizes (> 200) in stable, parasite-free environments for at least 50 generations prior to the coevolution experiment. Eight experimental beetle lines were set up, each as a unique combination of two stock populations (seven stock populations were used in total). Fifty virgin females from one stock line were crossed with fifty virgin males from the other, the reciprocal crosses were made with equal numbers, and all offspring were pooled to serve as the starter generation of the experimental line. We chose to set up our experimental lines in such a way because stock populations are likely to harbour reduced genetic diversity.

Subsequently, each line was split up in two treatments: coevolution and control. All eight lines were thus represented in both treatments. Corresponding lines (the same line in both the control and the coevolution treatment) were considered paired in the analyses, and consequently each given pair has the same genetic background. The lines in the coevolution treatment were subjected to selection by coevolving $N$. whitei, while the same lines in the control treatment were always kept and handled in identical ways, except that the medium was parasite-free and the hosts thus uninfected. $N$. whitei is a directly transmitted microsporidian parasite that is - from all what is known so far - reproducing asexually [42]. Every generation 500 unsexed beetles from the previous generation were used as breeders to initiate the next host generation. A mixture of eight different $N$. whitei isolates (to ensure sufficient standing genetic variation in the parasite population) was used to infect the first generation of beetles in the coevolution treatment, by mixing spores into the standard medium at a concentration of $2 * 10^{4}$ spores $\mathrm{g}^{-1}$ of medium. Every generation dead larvae harbouring $N$. whitei spores were collected, and a $N$. whitei powder was created by grounding and sieving the larvae. This was done for each experimental line separately. The next generation of each coevolving beetle line was then infected with their own unique mix of $N$. whitei spores derived from dead beetle larvae from the generation before. By selecting parasites that were able to infect and kill their hosts, and hosts that were able to survive the previous generation, it was ensured that both coevolving partners were exerting antagonistic selective pressures on each other [41]. Recombination frequency was estimated from beetles taken from the eleventh generation of the experiment.

\section{Recombination measurement}

Ten males from each line in both treatments were collected as pupae. Each reproductively mature male that emerged from these pupae was then crossed with one virgin female from an unrelated marker strain (strain LG1). Six (from the eight) experimental lines were chosen based on the success of the crosses, and eight males per line (from the ten that were crossed with a LG1 female) were selected in both the control and the coevolved treatment. These beetles were scored for 
Table 1 Primer characteristics of the used microsatellite markers (* derived from [44])

\begin{tabular}{|c|c|c|c|c|c|}
\hline Primer name & Linkage group & Position & Repeat & Forward primer & Reverse primer \\
\hline Tca-3.19* & 3 & 5940315 & AAT & CCATTGCAGATTGTAGGGTGT & GTTITACAGCGCCGAACAT \\
\hline LGIII2 & 3 & 6593045 & AAT & CATCACTTGGGTGCTITATCC & CAATACCTGAATGTGTGTGTGC \\
\hline LGIII3 & 3 & 9169070 & ATA & CACTATTTCCGCATATTGTTGC & TTATCCCTCTITGGCAGACG \\
\hline LGVI & 6 & 9124774 & TAA & CAAAGCACTCATGTACGAAACC & CCTCTTATTGACTTGTGTTATGACC \\
\hline Tca-6.11* & 6 & 5965031 & AAT & TAGTCTGCCGGCTGGTAAGT & AGCGACCGACATTTGTGTTT \\
\hline Tca-6.2* & 6 & 3982475 & A & TITIGTTGGGACACCCTGTA & TTGCGACGTATTTTCATTCG \\
\hline Tca-7.2* & 7 & 4598114 & A & GCTCGATTGGTAGGTGTGGT & AAAGCCTTTCACCTCCATTCT \\
\hline LGVIII & 7 & 904671 & AAT & TTGTCTCTITCAGGCCAAGG & GCTGAAATACTGGTCTGAGATGC \\
\hline LGVIII & 7 & 5833915 & $\mathrm{~A} T \mathrm{~T}$ & AAGGCATGCTTTGGTTCC & TGAATGCCGAAGACTAGTATGG \\
\hline Tca-10.1* & 10 & 4582080 & AAT & AAATTCTCGGCTITITGGGT & GAGCTGGCGGTTATATTGGA \\
\hline LGX4 & 10 & 8139860 & CGG & ATAGTTGCGCGCCTTTCG & ACATCACTGCGTCATGCTAGG \\
\hline
\end{tabular}

heterozygosity of 11 microsatellite markers (Table 1 ), distributed over four linkage groups (nrs. 3, 6, 7 and 10), using published methods [14].

For the molecular analyses, beetles from four control lines and their corresponding, paired coevolved lines were chosen. So in the end, only four of the eight experimental lines were used to score recombination. Initial choice of experimental lines was based on the availability (i.e. homozygous loci cannot reveal recombination events) and distribution (i.e. for a genome-wide coverage) of heterozygous loci within individuals. Hence, each line could have a different combination of diagnostic loci that border intervals of varying lengths. From each selected line, three males (from the eight that were scored for heterozygosity) were selected based on the same criteria of availability and distribution of loci, and up to 24 offspring per male were genotyped with the appropriate markers. Recombination rate was calculated as the number of recombinant offspring divided by the total number of screened offspring. A total of 576 offspring were genotyped to score recombination in 24 males (four lines, two treatments, three males per treatment per line), and recombination was successfully scored in 1'416 cases (meaning that recombination was scored in an average of about 2.5 interval per male).

The observed recombination frequency does not necessarily reflect the true recombination frequency, especially in larger genomic intervals [45]. Multiple numbers of recombination events will downward bias our estimate, as they cancel each other out and can result in what appears to be a non-recombinant genotype [46]. Besides double recombination events, also crossover interference could affect the observed recombination frequency. As crossover interference has been shown to occur in T. castaneum [30], we decided to use Kosambi's map function [47] to calculate map distances from our observed recombination frequencies. Kosambi's function corrects for both double recombination events and the occurrence of crossover interference [46].

\section{Expected map distance}

For each linkage group we acquired the genetic position (cM) and sequences of BAC (Bacterial Artificial Chromosome) ends and ESTs (Expressed Sequence Tag) that were used to create a genetic linkage map of $T$. castaneum [35]. We performed a BLAST (Basic Local Alignment Search Tool) search of the sequences against the $T$. castaneum genome [48] to get their physical position (bp). Per linkage group, genetic position was plotted as a function of physical position, and a fourth order polynomial was fitted through the points (SPSS 19 for Mac OS $\mathrm{X}$; all functions explained more than $98 \%$ of the variation). Knowing the physical position of the two markers bordering a given interval, we were able to calculate an expected map distance for each interval [23].

All experiments have been done in accordance with the regulations of ETH and Switzerland.

\section{Data analysis}

Linear regression (SPSS 19 for Mac OS X) was used to investigate several possible relationships. In both cases where we found a significant relationship, logarithmic trend lines $(\mathrm{y}=$ intercept + slope $* \ln (\mathrm{x}))$ provided a better fit than straight trend lines $(\mathrm{y}=$ intercept + slope $* \mathrm{x})$.

To be able to compare the slopes of the regression lines for the relationship between the mean relative difference between observed and expected map distance and the expected map distance of the control and the coevolution treatment (Figure 1), all expected map distances were $1 n$-transformed. The mean relative differences for both treatments were combined in one dataset, and the variables were defined as follows: y contains the difference values, $\mathrm{x} 1$ is a dummy variable to split up the data set in the two treatments $(0=$ coevolved, 1 = control), $\times 2$ contains the $\ln$-transformed expected map distances, and $\times 3$ is the product of $x 1$ and $\times 2$. Then a linear regression was performed using the following model: $y=\beta 0+\beta 1^{*} \times 1+\beta 2^{*} \times 2+\beta 3^{*} \times 3$. In this model $\beta 3$ represents the difference between the 
slopes of the regression lines of the two different treatments. It was tested if $\beta 3$ deviates significantly from zero.

\section{Acknowledgements}

We thank D. Heinzmann and T. Silber for help in the lab, and the Genetic Diversity Centre (GDC) at ETH for providing a work platform. This study was supported by grant nr. 31-120451 from the Swiss National Science Foundation to KMW.

\section{Author details}

${ }^{1}$ ETH Zürich, Institute of Integrative Biology, Experimental Ecology, $\mathrm{CH}-8092$ Zürich, Switzerland. ${ }^{2}$ University of Edinburgh, Institute of Evolutionary Biology, Edinburgh EH9 3JT, UK. ${ }^{3}$ Leibniz Institute for Marine Sciences (IfMGeomar), Evolutionary Ecology of Marine Fishes, Kiel, Germany. ${ }^{4}$ Wadden Sea Station Sylt, Alfred Wegener Institute for Polar and Marine Sciences, List/Sylt, Germany.

\section{Authors' contributions}

NAGK carried out the recombination measurements, analysed the data, and drafted the manuscript. CB carried out the coevolution experiment. PS and KMW conceived the study and coordinated it. All authors participated in the design of the study, read and contributed to the manuscript, and approve the final manuscript.

Received: 3 November 2011 Accepted: 13 February 2012

Published: 13 February 2012

\section{References}

1. Barton $\mathrm{NH}$ : A general model for the evolution of recombination. Genet Res 1995, 65(2):123-144.

2. Bell G: The Masterpiece of Nature: The Evolution and Genetics of Sexuality London: Croon Helm; 1982.

3. Hamilton WD: Sex versus non-sex versus parasite. Oikos 1980, 35(2):282-290.

4. Jaenike J: An hypothesis to account for the maintenance of sex within populations. Evol Theory 1978, 1:1-30.

5. Schmid-Hempel P: Evolutionary Parasitology Oxford: Oxford University Press; 2011.

6. Hamilton WD, Axelrod R, Tanese R: Sexual reproduction as an adaptation to resist parasites (a review). Proc Natl Acad Sci USA 1990, 87(9):3566-3573.

7. Haldane JBS: Disease and evolution. Ric Sci 1949, 19:68-76.

8. Peters $A D$, Lively $C M$ : The red queen and fluctuating epistasis: $A$ population genetic analysis of antagonistic coevolution. Am Nat 1999, 154(4):393-405.

9. Salathe $M$, Kouyos RD, Bonhoeffer $S$ : The state of affairs in the kingdom of the red queen. Trends Ecol Evol 2008, 23(8):439-445.

10. Salathe M, Kouyos RD, Regoes RR, Bonhoeffer S: Rapid parasite adaptation drives selection for high recombination rates. Evolution 2008, 62(2):295-300

11. Otto SP, Nuismer SL: Species interactions and the evolution of sex. Science 2004, 304(5673):1018-1020.

12. Agrawal AF: Differences between selection on sex versus recombination in Red Queen models with diploid hosts. Evolution 2009, 63(8):2131-2141.

13. Schulte RD, Makus C, Hasert B, Michiels NK, Schulenburg H: Multiple reciprocal adaptations and rapid genetic change upon experimental coevolution of an animal host and its microbial parasite. Proc Natl Acad Sci USA 2010, 107(16):7359-7364.

14. Bérénos $C$, Wegner KM, Schmid-Hempel P: Antagonistic coevolution with parasites maintains host genetic diversity: an experimental test. Proc $R$ Soc B Biol Sci 2011, 278(1703):218-224.

15. Koskella B, Lively CM: Evidence for negative frequency-dependent selection during experimental coevolution of a freshwater snail and a sterilizing trematode. Evolution 2009, 63(9):2213-2221.

16. Dybdahl MF, Lively CM: Host-parasite coevolution: evidence for rare advantage and time-lagged selection in a natural population. Evolution 1998, 52(4):1057-1066.

17. Wolinska J, Spaak P: The cost of being common: evidence from natural Daphnia populations. Evolution 2009, 63(7):1893-1901.
18. Lively CM, Dybdahl MF: Parasite adaptation to locally common host genotypes. Nature 2000, 405(6787):679-681.

19. Decaestecker E, Gaba S, Raeymaekers JAM, Stoks R, Van Kerckhoven L, Ebert D, De Meester L: Host-parasite 'Red Queen' dynamics archived in pond sediment. Nature 2007, 450(7171):870-873.

20. Jokela J, Dybdahl MF, Lively CM: The maintenance of sex, clonal dynamics, and host-parasite coevolution in a mixed population of sexual and asexual snails. Am Nat 2009, 174:S43-S53.

21. Lively CM, Craddock C, Vrijenhoek RC: Red queen hypothesis supported by parasitism in sexual and clonal fish. Nature 1990, 344(6269):864-866.

22. Camacho JPM, Bakkali M, Corral JM, Cabrero J, Lopez-Leon MD, Aranda I, Martin-Alganza A, Perfectti F: Host recombination is dependent on the degree of parasitism. Proc R Soc Lond B Biol Sci 2002, 269(1505):2173-2177.

23. Wegner KM: Clustering of drosophila melanogaster immune genes in interplay with recombination rate. PLOS One 2008, 3(7):8.

24. Lucht JM, Mauch-Mani B, Steiner HY, Metraux JP, Ryals J, Hohn B. Pathogen stress increases somatic recombination frequency in Arabidopsis. Nat Genet 2002, 30(3):311-314.

25. Baer B, Schmid-Hempel P: Experimental variation in polyandry affects parasite loads and fitness in a bumble-bee. Nature 1999, 397(6715):151-154

26. King KC, Lively CM: Geographic variation in sterilizing parasite species and the red queen. Oikos 2009, 118(9):1416-1420

27. Lively CM: Evidence from a New Zealand snail for the maintenance of sex by parasitism. Nature 1987, 328(6130):519-521.

28. Morran LT, Schmidt OG, Gelarden IA, Parrish RC, Lively CM: Running with the red queen: host-parasite coevolution selects for biparental sex. Science 2011, 333(6039):216-218.

29. Fischer O, Schmid-Hempel P: Selection by parasites may increase host recombination frequency. Biol Lett 2005, 1(2):193-195.

30. Greeff M, Schmid-Hempel P: Influence of co-evolution with a parasite Nosema whitei, and population size on recombination rates and fitness in the red flour beetle, Tribolium castaneum. Genetica 2010, 138(7):737-744.

31. Blaser M, Schmid-Hempel P: Determinants of virulence for the parasite Nosema whitei in its host Tribolium castaneum. J Invertebr Pathol 2005, 89(3):251-257

32. Wegner KM, Berenos C, Schmid-Hempel P: Nonadditive genetic components in resistance of the red flour beetle Tribolium castanaeum against parasite infection. Evolution 2008, 62(9):2381-2392.

33. Wegner KM, Berenos C, Schmid-Hempel P: Host genetic architecture in single and multiple infections. J Evol Biol 2009, 22(2):396-404.

34. Otti O: Host-parasite interactions - experimental studies on virulence and transmission. PhD thesis ETH Zürich; 2007

35. Lorenzen MD, Doyungan Z, Savard J, Snow K, Crumly LR, Shippy TD, Stuart JJ, Brown SJ, Beeman RW: Genetic linkage maps of the red hour beetle, Tribolium castaneum, based on bacterial artificial chromosomes and expressed sequence tags. Genetics 2005, 170(2):741-747.

36. Otto SP, Barton NH: Selection for recombination in small populations. Evolution 2001, 55(10):1921-1931.

37. Stevens L, Yan GY, Pray LA: Consequences of inbreeding on invertebrate host susceptibility to parasitic infection. Evolution 1997, 51(6):2032-2039.

38. Bérénos C, Schmid-Hempel P, Wegner KM: Experimental coevolution leads to a decrease in parasite-induced host mortality. J Evol Biol 2011 24(8):1777-1782

39. Dewees AA: Genetic modification of recombination rate in Tribolium castaneum. Genetics 1975, 81(3):537-552.

40. Kraaijeveld AR, Godfray HCJ: Trade-off between parasitoid resistance and larval competitive ability in Drosophila melanogaster. Nature 1997, 389(6648):278-280

41. Bérénos C, Schmid-Hempel P, Wegner KM: Evolution of host resistance and trade-offs between virulence and transmission potential in an obligately killing parasite. J Evol Biol 2009, 22(10):2049-2056.

42. Ironside JE: Multiple losses of sex within a single genus of microsporidia. BMC Evol Biol 2007, 7:16.

43. Ebert D: Host-parasite coevolution: Insights from the Daphnia-parasite model system. Curr Opin Microbiol 2008, 11(3):290-301.

44. Demuth JP, Drury DW, Peters ML, Van Dyken JD, Priest NK, Wade MJ: Genome-wide survey of Tribolium castaneum microsatellites and description of 509 polymorphic markers. Mol Ecol Notes 2007 7(6):1189-1195. 
45. Haldane JBS: The combination of linkage values, and the calculation of distances between the loci of linked factors. J Genet 1919, 8(4):299-309.

46. Huehn M: On the bias of recombination fractions, Kosambi's and Haldane's distances based on frequencies of gametes. Genome 2011, 54(3):196-201.

47. Kosambi DD: The estimation of map distances from recombination values. Ann Eugen 1944, 12(3):172-175.

48. BeetleBase website. [http://www.beetlebase.org].

doi:10.1186/1471-2148-12-18

Cite this article as: Kerstes et al: Antagonistic experimental coevolution with a parasite increases host recombination frequency. BMC

Evolutionary Biology 2012 12:18.

Submit your next manuscript to BioMed Central and take full advantage of:

- Convenient online submission

- Thorough peer review

- No space constraints or color figure charges

- Immediate publication on acceptance

- Inclusion in PubMed, CAS, Scopus and Google Scholar

- Research which is freely available for redistribution

Submit your manuscript at www.biomedcentral.com/submit 